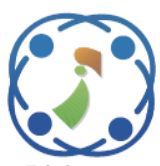

\title{
A New Approach to Predict Potential Sleep Disorder based on Fractal Analysis from Non-overlapping Single Lead ECG Using Support Vector Machine
}

\author{
Iman Fahruzi ${ }^{1,2}$ \\ I Ketut Eddy Purnama ${ }^{1,3,4}$ \\ Kayo Yoshimoto 5 \\ Hideya Takahashi ${ }^{5}$ \\ Mauridhi Hery Purnomo ${ }^{1,3,4 *}$ \\ ${ }^{I}$ Department of Electrical Engineering, Faculty of Intelligent Electrical and Informatics Technology, \\ Institut Teknologi Sepuluh Nopember, Surabaya, Indonesia \\ ${ }^{2}$ Department of Electrical Engineering, Politeknik Negeri Batam, Indonesia \\ ${ }^{3}$ Department of Computer Engineering, Faculty of Intelligent Electrical and Informatics Technology, \\ Institut Teknologi Sepuluh Nopember, Surabaya, Indonesia \\ ${ }^{4}$ University Center of Excellence on Artificial Intelligence for Healthcare and Society (UCE AIHeS) \\ ${ }^{5}$ Department of Electrical and Information Engineering, Osaka City University, Osaka, Japan \\ * Corresponding author's Email: hery@ee.its.ac.id
}

\begin{abstract}
Sleep disorders are challenging to diagnose. The complexity of records obtained from electrocardiogram (ECG) recordings requires manual inspection by experienced medical practitioners. Meanwhile, ECG records are still widely used to diagnose heart problems during sleep. To resolve the issue, the fractal analysis is a promising means to help identify the characteristics of non-overlapping apnea and non-apnea events based on signal scaling behaviour and QRS wave morphologies. Therefore, we propose a new approach to develop automatic sleep disorder classification to minimalize visual inspection and manual scoring. We employed the monofractal and the multifractal analyses to generate new features such as alpha1, residue1, alpha2, residue2, Dqmin, Dqmax, hqmin, hqmid, hqmax, and hqmaxhqmin. To improve the proposed method's performance, we used the ten new features that have been extracted, which are eventually being used as inputs space to a support vector machine (SVM). Through examining the feature set, we designed an optimum SVM model classifier to explore the usability of patterns to predict potential sleep disorder corresponding to apnea and non-apnea events. Hence, our approach through SVM with radial basis function (RBF) kernel is achieved to have accuracy, sensitivity, specificity of $92.16 \%, 88.24 \%, 94.12 \%$ respectively.
\end{abstract}

Keywords: Fractal analysis, Multifractal, Sleep disorder, SVM, Fractal scaling behaviour.

\section{Introduction}

Sleep is one of the human rest phases. Sleep quality has an impact on freshness and normality of the human organs. Therefore, it is crucial to maintain sleep quality. The development of technology and modern lifestyle make people pay less attention to health and quality ways of life. In the long term, the quality of life, to some extent, will be determined by sleep behaviour. Obstructive sleep apnea-hypopnea syndrome (OSAHS) is a chronic sleep disorder condition. OSAHS is characterized by a recurrent recurrence of the respiratory tract so that people often wake up throughout the night. These conditions make International Journal of Intelligent Engineering and Systems, Vol.14, No.2, 2021 the body condition during the day becomes weak. Further, these conditions can lead to a more severe problem in the human heart. The most severe conditions will cause hypertension, stroke and other heart problems [1]. Sleep problems may be treated and diagnosed individually or in the hospital.

For the initial screening, a polysomnography (PSG) is a useful and gold standard for comparison by the hospital. However, there are weaknesses such as the element of subjectivity in performing evaluations that is qualitative and rely heavily on assessment experience. Naturally, varying levels of experience contribute to various evaluations. In addition, there can also be variations in diagnosis due 
to human errors and the presence of human interference in visual inspection. PSG usually consists of several electrodes. During test, many wires and channels are attached on the persons of body. These conditions are very complicated because of the comprehensive monitoring of the many physiological signals.

Studies on biological signals for the overview of sleep disorders include the use of Heart Rate Variability (HRV) [2, 3]. Other studies use RR interval to identify slow wave sleep from ECG [4]. Another method to detection OSA using an optimal orthogonal wavelet filter bank with ECG signals [5]. This technique has been utilizing the morphology of the pQRSt waves from the ECG signal to acquire four groups of feature sets to identify the severity of Obstructive Sleep Apnea (OSA) [6]. Another approach uses a variation between normal patients and epilepsy patients based on HRV using timevarying autoregressive modelling [7]. In addition, the features of intrinsic band functions come from ECGDerived Respiration (EDR) Signals and HRV measurements [8]. The feature value is obtained from Q wavelet to sub bandwidth ECG signals [9].

In order to obtain the best results, an analysis of the acquisition and preprocessing stages of the signal was needed. However, the analysis should not damage the characteristics of the signal. In addition, the process is without eliminating the desired clinical information. Several studies related to ECG signals preprocessing include new method for ECG baseline wander removal using empirical mode decomposition [10]. Moreover, signal wandering is very influential on the results of the extraction process. A different approach using Hilbert transform to eliminate baseline wander ECG signals has also been performed [11]. Some classification algorithms with various methods for detecting sleep disorders were also commenced several years ago, including classifying five cases in sleep disorders using support vector machine [2]. Feature extraction from ECG to obtained inter beat intervals (IBIs) and classify sleep stage using A long short-term memory (LSTM) [12] and other classifiers approach using modern machine learning to classify OSA [13].

Another research classifies normal and apnea variations, based on the features of spectral analysis and the statistical value of ECG signal, using the bootstrap aggregation classifier [14]. Moreover, another method to detect sleep apnea uses RUSboosted based on the feature set of the UnableQ factor wavelet transform [15]. However, there are some limitations to these previous studies. These approaches did not reconstruct the complexity of ECG signals as a result of degradation during data acquisition and preprocessing stages. In addition, the ectopic beats, arrhythmic events, missing data and noise effects, may lead to the corrupt data along with the QRS interval and there are irregularities in the absolute and relative values of the frequency band. Therefore, the potential loss of clinical information is significant. The deviation of potential loss from the following requirements may lead to unpredictable results that are difficult to interpret. The lost clinical information leads to difficulties and errors in the diagnosis of signal patterns in patients with sleep disorders. Therefore, a tool is needed for a more indepth analysis of ECG morphology, QRS intervals and periodic patterns of ECG signals to get the difference between apnea and non-apnea signalling patterns.

The conventional method of visual scoring is still the most acceptable approach to date. However, it involves the interpretation of the various signals by visual data. Automatic scoring is, therefore seen as an effective approach and minimizing manual inspection for human error prevention. The complexity and trending signals in the ECG recording reflect the mechanisms of physiology as the heart functions. The occurring dynamics can be studied very accurately using the process of fractal analysis. Throughout this research, we merged two methods from nonlinear fractal analysis to obtained the fractal scaling behaviour features that correspond to the ECG signal pattern throughout patients with sleep disorders. These approaches are monofractal analysis to obtain the fluctuation function of signal and multifractal analysis for spectrum multifractal. The combination of these methods used to obtain ten features. In order to make the most of the training and testing process, the assessment scenario is conducted in a variety of approaches, such as identifying the suitable method for feature extraction, setting the parameters to develop the appropriate model, and evaluating the system using all of the features. To achieve an appropriate diagnosis and treatment based on different fractal scaling signal behaviour and QRS morphology phenomenon, precise sleep scores are deemed to be the critical part of the process.

The contributions of this study are: (1). The investigation of ECG recordings robustness and complexity is only from single lead ECG with a duration of more than ten-minute segments; (2). Employing a smaller number of features to obtain a significant advantage for the classification by decreasing the time of training and testing; (3). The screening process of ECG to obtain acceptable pattern distinctions between apnea and non-apnea is based on signal behaviour and QRS morphologies using multifractal analysis; (4). Applying the 
optimization parameters to the automatic correction of hyperplane using the various kernel of SVM; (5). To develop real-time classification sleep disorder to minimalize visual inspection and manual scoring by the medical employee.

This paper is structured as follows. We describe the sleep disorder, previous study and related work in Section 1. Section 2 describes the proposed method to predict potential sleep disorder based on fractal analysis. Section 3 describes the experimental results and analysis for local trending based on detrended fluctuation analysis (DFA) and multifractal spectrum based on multifractal detrended fluctuation analysis (MFDFA). Section 4 discussed our approach and compared with other works. Finally, the conclusion of this study provided in Section 5.

\section{Data description and methods}

\subsection{ECG-apnea recordings}

In this study, the physionet database provides the dataset used in the experiment [16]. The recordings were an acquisition at a sampling rates of $100 \mathrm{~Hz}$ and 16-bit resolution from single-lead ECG. The recording was scored by the clinical experts. This study used 502 non-overlapping recordings with an average duration of 13.10 minutes in length. The distribution of recording is $61.75 \%$ of apnea events and $38.25 \%$ of non-apnea events. To measure the performance of the proposed framework, the nonoverlapping ECG signals are divided into three categories, training set, validation set and testing set. Fig. 3 (a) shows the non-overlapping segmented signals, non-apnea event and apnea event.

\subsection{Preprocessing of ECG-apnea recordings}

The ECG-apnea recordings from the physionet database have a very long duration ranging from 7 to 8 hours. The ECG recording was preprocessing to improve the quality of the signal. We used some methods to elimination noise, namely WA detrend method to removing baseline wander signal and undecimated wavelet transform (UWT) method to noise suppression [17]. The recordings of ECG have robust heart rate variability. If the heart rhythm is normal, the heart rate can be measured by the interval between two consecutive R waves as shown in Fig. 1 . Disturbance of small spike is reducing long-term HRV. These condition takings changes of RR intervals while comparing successive values of $R$ beats.

Therefore, perfection in preprocessing is a critical phase. In order to extract ECG signal, it must be free

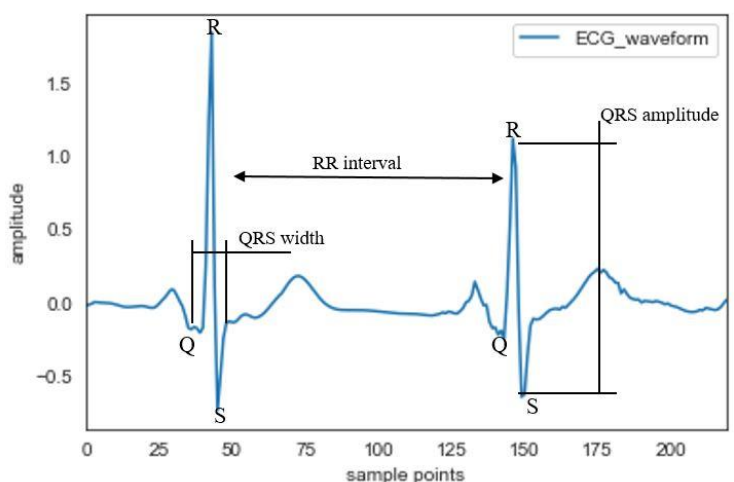

Figure. 1 ECG waveform, QRS morphologies and various of RR interval
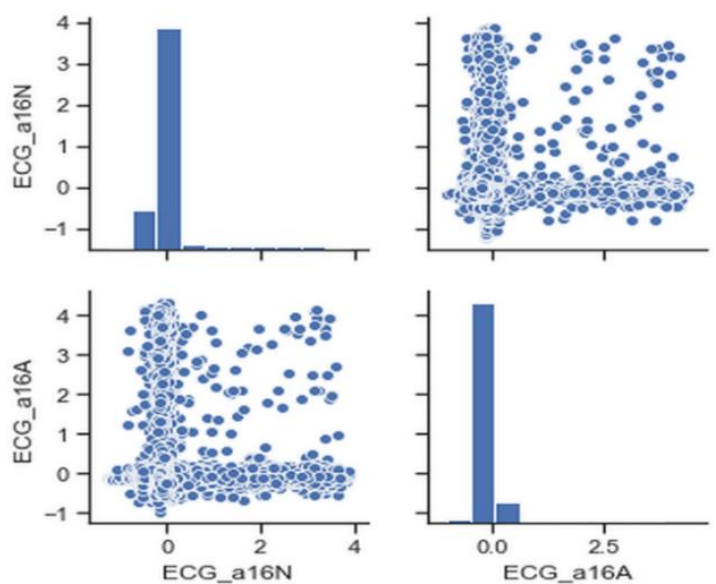

Figure. 2 An example of the graph distribution of data point for apnea event and non-apnea event

from signal damage or noise interference. In case, this is not done carefully, it will cause a signal to lose clinical information. The loss means that clinical experts will have difficulty diagnosing. An incomplete diagnosis will harm the patient because of erroneous medical treatment. The removed contaminating noise is shown in Fig. 3 (b). In this process, the signal will be reconstructed to eliminate some effects of noise, such as the effect of power line interference noise, the effect of medical equipment noise and other influences that make the morphological quality of the ECG signal imperfect. The ECG signal imperfection caused by the noise will significantly affect the detection process of ECG waves.

Fig. 2 shows the graph distribution of data point for apnea event, ECG_a16A (the first column) and non-apnea event, ECG_a16N (the second column). It can be described that the data points are mapped in the form of a scatterplot and histogram to clearly define the difference based on the relationship and similarity of the paired relationship in the dataset. Moreover, the disparity in distribution patterns is an efficient input in the implementation of the machine 


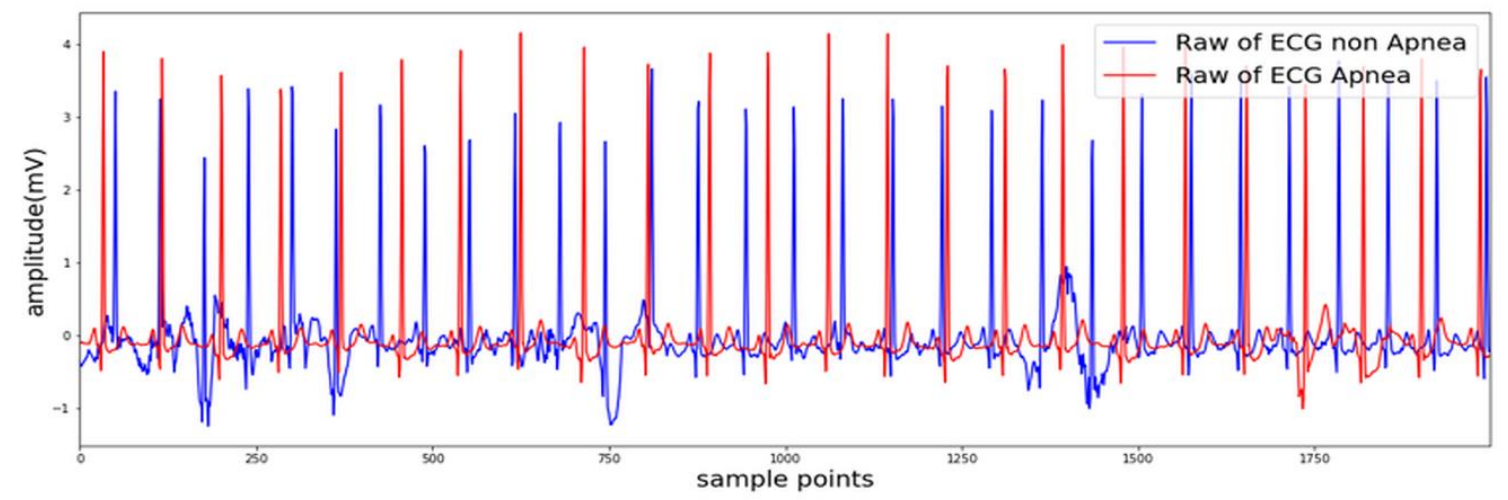

(a)

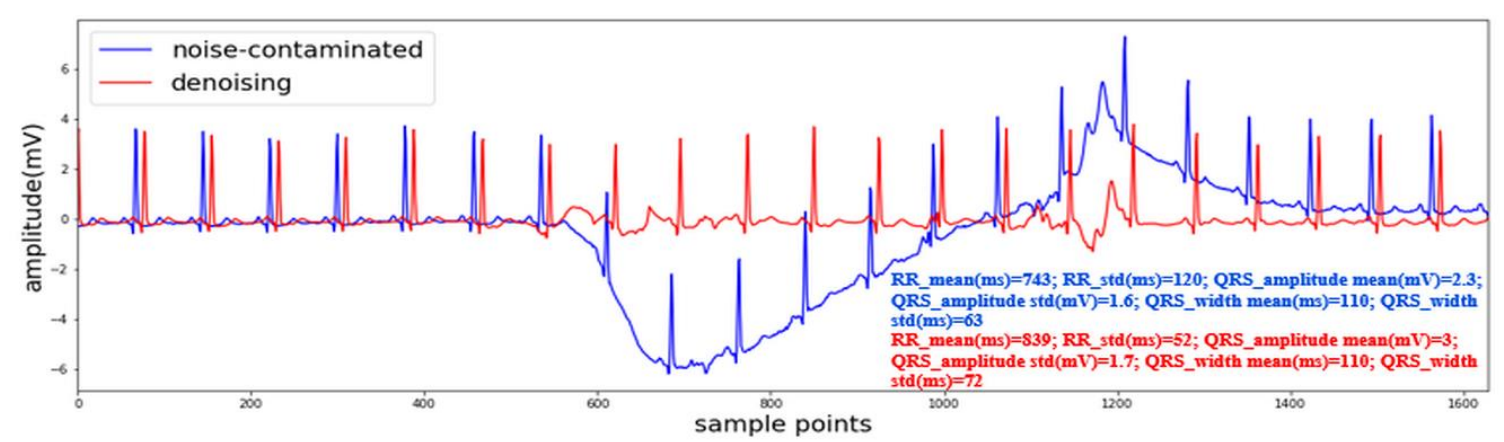

(b)

Figure. 3 The representation of ECG signals: (a) the observed raw data of ECG apnea and non-apnea events and (b) the contaminating noise and denoising of the ECG

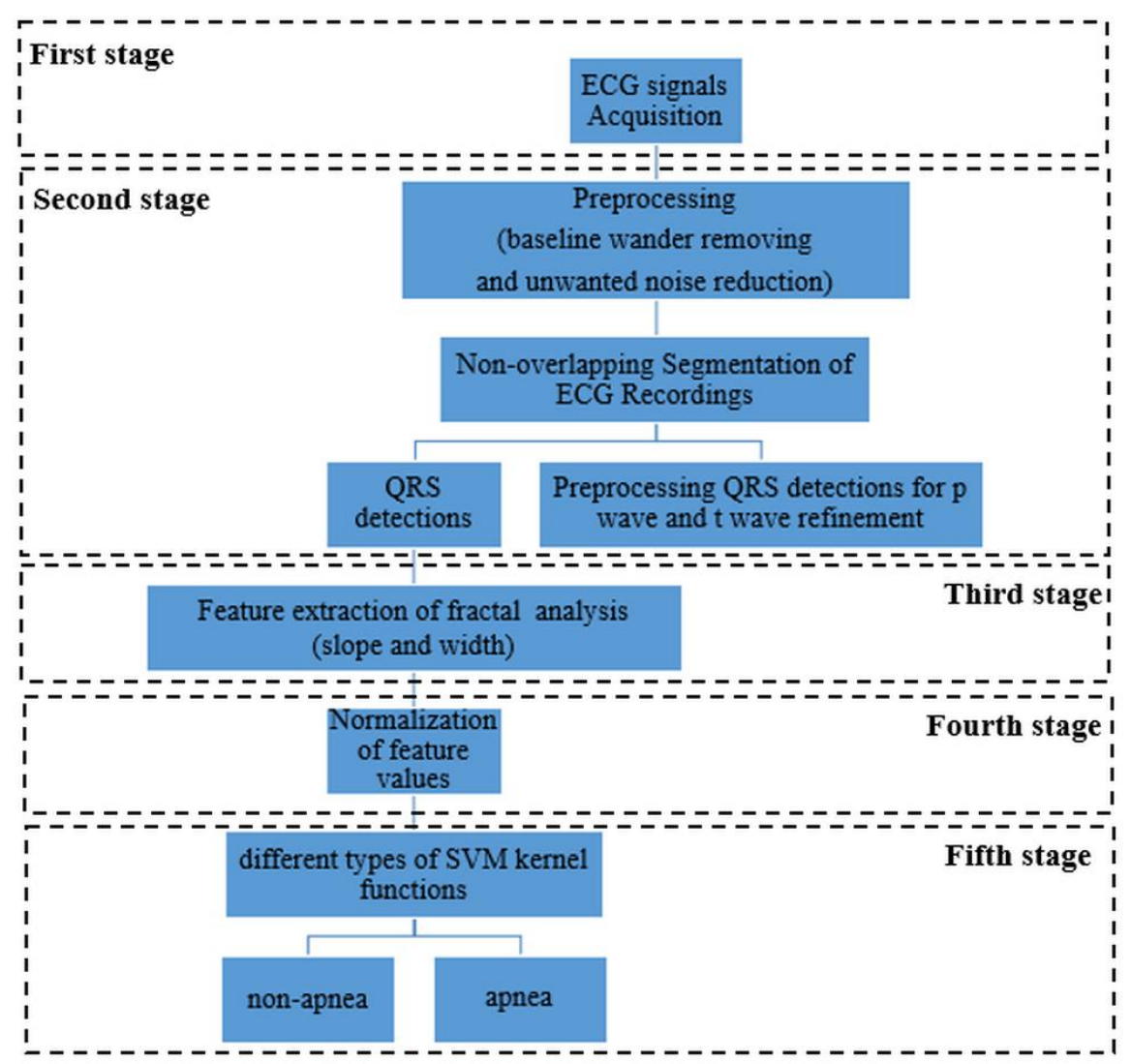

Figure. 4 Block diagrams of the proposed method 
learning. The following features value have been provided based on RR interval and QRS morphologies characteristics for apnea and nonapnea events. The non-apnea event has heart rate mean of 82 beats per minute (BPM) and heart rate standard deviation of 6.8 BPM through the length of 1523 beats. The apnea event has heart rate mean of $72 \mathrm{BPM}$ and heart rate standard deviation of 4.8 BPM through the length of 997 beats. Moreover, a further in-depth investigation is required to identify the differences in the characteristics of the QRS complex based on changes in the adjacent $\mathrm{R}$ waves. Based on the results of this study, the apnea ECG recording contains RR mean of 743 milliseconds, RR standard deviations of 120 milliseconds, QRS amplitude mean of 2.3 millivolts, QRS amplitude standard deviations of 1.6 millivolts, QRS width mean of 110 milliseconds, and QRS width standard deviations of 63 milliseconds. Conversely, a non-apnea recording has RR mean of 839 milliseconds, RR standard deviations of 52 milliseconds, QRS amplitude mean of 3 millivolts, QRS amplitude standard deviations of 1.7 millivolts, QRS width mean of 110 milliseconds, and QRS width standard deviations of 72 milliseconds.

Furthermore, the comparison of each feature with p-value is computed based on raw data ECG recordings and observed statistic distribution. These logarithmic deviations are used to obtain features with more rapid to normal distributions. Based on the quantitative evaluation, the data distribution characteristics comparison shows that there is a significant difference in the pattern of the ECG signals associated with any changes that occurs as a result of periodic failures and unexpected construction. As can be observed from Table 1, it was found that the features with $p$-values lower than 0.05 can be used to differentiate between apnea and nonapnea. These features are alpha1, alpha2, residue2, hqmin, hqmid, Dqmax, and hqmaxhqmin. The significant level was very useful to optimize the classifier and improve the performance parameters.

The block diagram proposed in this work is as shown in Fig. 4. In the first stage, the ECG signal was acquired through the annotation that has been validated by the medical expert to get the apnea signal and the non-apnea signal. Then, in the second stage, the preprocessing of ECG recordings to automatically remove noise interference was employed to deduce baseline wander and noise elimination. These schemes were used to ensure there was no interference that would potentially disturb QRS waves detection based on RR interval extraction. Henceforth, the segmentation process was carried out without overlapping noticed by an expert. In addition, preprocessing and QRS detection implies to the segmented signal refinement by adjusting QRS frequency range, QRSonset, QRSoffset, and bandpass filter setting for fiducial point of other waves, i.e., $p$ wave and $t$ wave. In the third stage, features are extracted from fractal analysis to get features that had similarities with the ECG signal pattern of patients with sleep disorders. In addition, features were obtained from a variety of techniques for an in-depth investigation, one of which was by calculating the statistical value of QRS morphologies, including QRS amplitude and QRS width. These parameters can identify whether there are differences in mean and standard deviation between apnea and non-apnea events. In the fourth stage, the normalization process was involved for all data to transform a specific range of feature value to meet machine learning requirement. These methods that result in features were further continued to reduce feature complexity due to the parts that were not relevant to the sleep disorder signal pattern and eliminate bias of unbalanced dataset. The fifth stage was to classify the collection of features into two classes, i.e., the class whose features have similarities with the signal pattern for apnea and the other classes which had similarities with the signal pattern for nonapnea.

\subsection{Feature extraction process using fractal analysis for apnea and non-apnea ECG signals}

In this section, the main purpose of the new proposed approach is an assessment of best practice to combine the fractal structure based on a short and long ranges of sleep disorder ECG signals using the feature set from fractal analysis methods. The fractal analysis aims to determine the periodic pattern of a signal at a certain period so that the fractal structure can be recognized based on variations in signal fluctuations. Recognition of biomedical signal patterns using the fractal structure approach provides another point of view for differences in patterns between the two classes.

It is noted that biomedical signals are essentially visible, but important information such as the amplitude and width of the QRS complex is complicated to capture if only employing conventional methods. The biomedical signals also have a very high degree of invariant scale and are continuously repeated. Therefore, it is efficient to analyse various physiological signals using the spectrum analysis method, specifically signals with an indication of sleep disorders. 
Table 1. The distribution feature value and significance level for apnea and non-apnea events

\begin{tabular}{|c|c|c|c|c|c|}
\hline Features & $\begin{array}{c}\text { Non-apnea } \\
\text { (mean and } \\
\text { standard } \\
\text { deviation) }\end{array}$ & $\begin{array}{c}\text { Apnea } \\
\text { (mean and } \\
\text { standard } \\
\text { deviation) }\end{array}$ & $\begin{array}{c}\text { Feature scaling of } \\
\text { Non-apnea (mean } \\
\text { and standard } \\
\text { deviation) }\end{array}$ & $\begin{array}{c}\text { Feature scaling of } \\
\text { Apnea (mean and } \\
\text { standard } \\
\text { deviation) } \\
\end{array}$ & p-value \\
\hline \multicolumn{6}{|c|}{ Monofractal feature set: } \\
\hline 1. Alpha1 & $0.93(0.27)$ & $1.25(0.33)$ & $0.57(0.77)$ & $0.36(0.92)$ & $<0.0001$ \\
\hline 2. Residue1 & $0.0005(0.0007)$ & $0.0005(0.0006)$ & $0.02(1.08)$ & $0.01(0.95)$ & $\geq 0.05$ \\
\hline 3. Alpha2 & $0.70(0.25)$ & $0.47(0.23)$ & $0.55(0.93)$ & $0.34(0.88)$ & $<0.0001$ \\
\hline 4. Residue2 & $0.0012(0.0012)$ & $0.0007(0.0008)$ & $0.32(1.19)$ & $0.20(0.80)$ & $<0.0001$ \\
\hline \multicolumn{6}{|c|}{ Multifractal feature set: } \\
\hline 5. Dqmin & $-170.8(2359.3)$ & $-476.7(6019.9)$ & $0.04(0.47)$ & $0.02(1.22)$ & $\geq 0.05$ \\
\hline 6. hqmin & $1.38(0.24)$ & $1.48(0.25)$ & $0.24(0.95)$ & $0.15(1.00)$ & $0.01-0.05$ \\
\hline 7. hqmid & $0.44(0.11)$ & $0.49(0.07)$ & $0.32(1.12)$ & $0.20(0.86)$ & $0.001-0.01$ \\
\hline 8. Dqmax & $0.69(0.32)$ & $0.64(0.69)$ & $0.05(0.57)$ & $0.03(1.19)$ & $0.001-0.01$ \\
\hline 9. hqmax & $0.10(0.13)$ & $0.09(0.13)$ & $0.05(0.99)$ & $0.03(0.99)$ & $\geq 0.05$ \\
\hline 10. hqmaxhqmin & $1.31(0.26)$ & $1.41(0.28)$ & $0.23(0.94)$ & $0.14(1.01)$ & $0.01-0.05$ \\
\hline
\end{tabular}

\subsection{Feature extraction using detrended signal analysis approach}

Generally, the biomedical signals with a dynamic invariant dimension, frequently neglect tiny or robust sections. Meanwhile, these small parts are often only described in the form of calculating the average value, standard deviation, maximum value, minimum value, and median value. This approach is not enough, especially for observations on signals of very long duration. Therefore, another approach is needed that can describe a signal through fractal structure analysis and to calculate the estimated low-exponent power as a representation of the scale invariant structure of the biomedical signal. The Monofractal analysis technique, or more well known as detrended fluctuation analysis (DFA), is used to analyze time series data [17-19]. The DFA approach is strongly related to the value of approximation error and successively attained the value of $F(n)$ as a function of $n$ and the value of alpha ( $\alpha$ ) as a slope (hq) value. In order to obtain a slope, we apply the following DFA approach:

1) Define the profile of signal $y(i)$ of length $\mathrm{N}$ :

$$
y(i)=\sum_{k=1}^{i}\left(R R_{(k)}-R R(\text { ave })\right), i=1, \ldots, N
$$

$R R_{(k)}$ is time series of length $N$, and $R R($ ave) is mean.

2) Calculate the local trend by a least square fit, and and determine variance of local trend segment $v, v=1$, ..., $N_{t}$. However, before that divide the profile $y(i)$ into $N_{t} \cong \operatorname{int}(N / t)$ non-overlapping segments of the equal duration as time scale $t$.

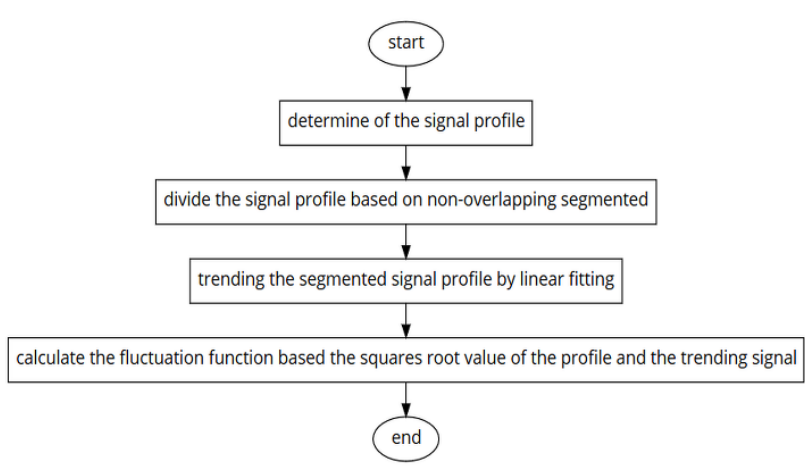

Figure. 5 The flow chart for calculating fractal scaling based monofractal analysis

$$
F_{t}^{2}(v) \cong \frac{1}{t} \sum_{i=1}^{t}\left[y((v-1) t+i)-p_{v}(i)\right]^{2}
$$

The parameter $p_{v}(i)$ is fitting process in the segment $v$.

3) Root-mean-square is calculated by:

$$
F(t) \cong\left[\frac{1}{2 N_{t}} \sum_{v=1}^{2 N_{t}} F_{t}^{2}(v)\right]^{1 / 2}
$$

Where $F(t)$ is fluctuation function. Furthermore, repeat procedure 2 and 3 to obtain the $\log F(n)$ and $\log n$ correlations as shown in Fig. 7 (a) and Fig. 7 (b).

Fig. 5 presents the flow chart for the process to determine signal slope and demonstrate the DFA approach to analysis self-similarity for short-range ECG-apnea signal.

As explained previously, the resulting slope has a statistical meaning. Therefore, through this monofractal analysis, the correlation between raw signal and local trending can be correlated. The 
results of the calculation will mean correlated for long range structure if the value of the slope is $0.5-1$, on the contrary to the slope $0-0.5$ means anticorrelated [21] for short range structure.

\subsection{Feature extraction using multifractal detrended signal analysis approach}

The monofractal analysis seems to be clarified earlier in order to detect a signal fractal structure, not only to obtain the slope of a signal but also to obtain certain parameters in the context of a multifractal width structure. The ECG signals that are invariant on the fractal structure scale are closely related to the changes that occur due to impulses from the heart while working. The process for the multifractal analysis stage is schematically illustrated in Fig. 6. The use of multifractal detrended fluctuation analysis (MFDFA) in biomedical time and heart rate analyses is useful for estimating the low exponent power of a signal [21-22]. The feature extraction from electroencephalogram (EEG) signals time series [23]. The time series analysis of daily temperature [24]. The leaf image feature analysis using multifractal analysis [25].

The multifractal parameters in the form of slope and width are extremely helpful in certain studies in the analysis or determination of signal differences. In order to achieve features that are important to the classifiers, the study of the ECG records using the multifractal spectrum to detect subtle morphology changes and heart rate variability expressed in width parameters will be proposed. As mentioned previously, the monofractal parameter is calculated by Eq. (1) and Eq. (2), while fluctuation function by Eq. (3). These processes are the basis of the experiment by multifractal analysis. In order to determine $F(t)$ how depends on the time scale $t$, it is obvious that $F(t)$ is increasing with $t$, since with larger segments the variations from the fits become bigger as fluctuation functions $F(t)$ in Eq. (4).

$$
F(t) \sim t^{h(q)}
$$

Whereas $h(q)$ is slope, $t$ is time scale. In the DFA approach, $h q$ parameter does not depend on changes in scaling exponent $q$. However, the $h q$ parameter of MFDFA process is affected by the value of $q$ because of the nature of $F_{t}^{2}(v)$ scaling behaviour for all values of $q$. The equations to generate the multifractal spectrum are defined to calculate the fractal dimension $(\mathrm{Dq})$, the following steps are implemented [22] as follow.

$$
t(q)=q h(q)-1
$$

Whereas, $t(q)$ is multifractal scaling exponent and $h(q)$ is slope. The fractal dimension $D(q)$ can be obtained by Eq. (6).

$$
D(q)=\frac{t(q)}{q-1}=\frac{q h(q)-1}{q-1}
$$

As revealed previously, the width parameter is represented by several related features, i.e., the fractal dimension (Dq) and slope (hq). Based on the MFDFA approach in time series [22], the Dq parameter is obtained based on the conversion of slope (hq) to the multifractal scaling exponent (tq) in the monofractal analysis by Eq. (6). Consequently, every change in the parameter $q$ will affect the fractal dimension (Dq).

\subsection{Various kernel of Support vector machine (SVM) classifier}

In this study, we used four type kernels of SVM to evaluate the model. The model is tested using polynomial kernel, sigmoid kernel, linear kernel and SVM RBF kernel. Particularly, these kernels are used to find an optimal hyperplane that separates the data point with the minimum error. The basic knowledge about the kernel is using the following equations [26]: linear kernel is denoted by Eq. (7), polynomial kernel is formulated by Eq. (8), RBF kernel is formulated by Eq. (9), and sigmoid kernel is formulated by Eq. (10).

$$
\begin{gathered}
K(x, u)=x^{T} u \\
K(x, u)=\left(a x^{T} u+c\right)^{q}, q>0 \\
K(x, u)=\exp \left(-\gamma\|x-u\|^{2}\right) \\
K(x, u)=\tanh \left(\beta x^{T} u+\gamma\right)
\end{gathered}
$$

Where $c$ is the regularization parameter to control the trade-off between margin and error of classification, $q$ is a degree of the polynomial kernel function to enable a more compact decision boundary, and $\gamma$ is gamma parameter as independent term to generate the sensitivity to differences in feature vectors.

\section{Results}

In this section, we elucidate the details of our experiments, present the results and discourse about their advantages.

\subsection{Analysis of local trending based DFA}

The local trending signal is basically to obtain the feature value of slope and feature value of residue fluctuation for each segment. These slope values are 


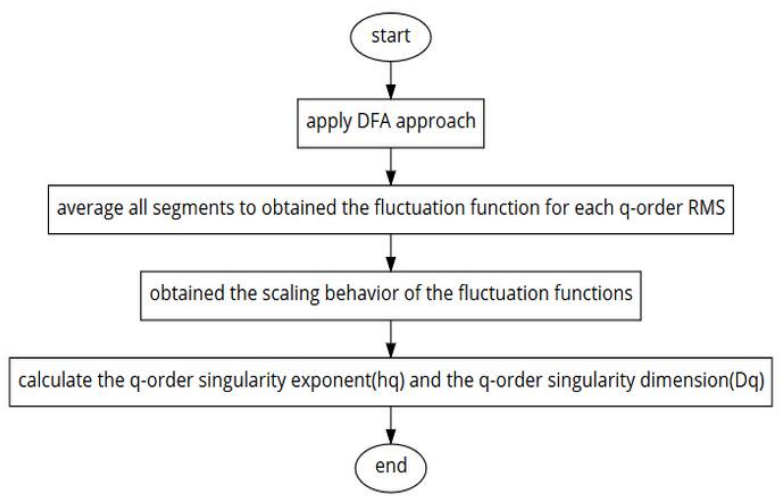

Figure. 6. The flow chart for calculating multifractal scaling exponent of MFDFA

the result of fitting in the raw signal profile based on the regression line. The slope value is represented in the alpha1 and residue 1 parameters which represent the results of the first fitting. In the other part, the results of the second fitting are represented in the form of alpha 2 and residue 2 parameters. Fig. 7 (a) shows the result of the local trending process on the apnea indication signal with a segment duration of 10 minutes 40 seconds (64090 data points). The results of the fitting in the apnea indication profile using the DFA analysis show the feature value of slope with the alpha1 parameter of 1.336 and residue 1 value of 0.00067 . Meanwhile, for the results of the second fitting in the profile, the nonlinear parameters show a range of slope value, i.e., alpha2, residue 2 of 0.15 , 0.00016 , respectively. As can be seen in the graph in
Fig. 7 (b) shows the results of local trending on the signal profile with a non-apnea indication. The profile length is in 8 minutes 40 seconds and the number of sample 52090 data points. It is clear from the figure that the result shows the parameters slope alpha1, residue 1 of $0.695,0.00049$, respectively. In addition, the result of the second fitting shows slope of alpha2, residue 2 of $0.64,0.00179$ respectively. The short-long scaling of the ECG signal is represented to the slope of the line. The alphal is an indicator for the short duration of the ECG signal based on the local trend of the signal result. However, for the long duration of the ECG signal based on the local trend of the signal result, alpha2 is the observed indicator. The slope value of more than 0.5 means that the signal has a similarity with the original signal. In other words, it has the smallest error value. The main difference between these measures to identify apnea characteristic from the non-apnea event. The root squares of the differences between the profile and the fits are used to calculate the fluctuation function in step 3 of the DFA approach. Therefore, the experimental results show that the trending profile will produce a large slope so that any changes due to fluctuations in the signal will affect the overall root mean square (RMS) error. The experiment results confirm that the monofractal analysis through the investigation of signal pattern behavior is better than the conventional approaches.

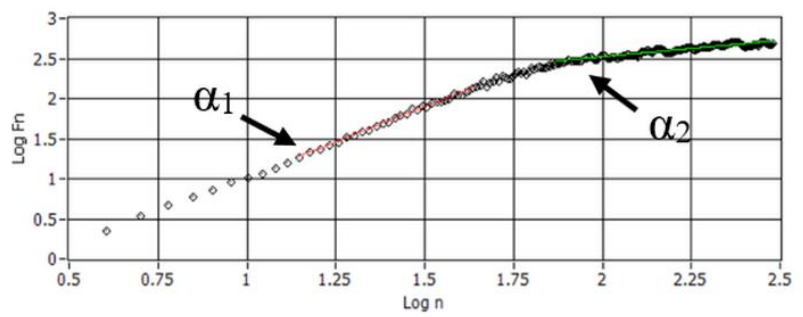

(a)

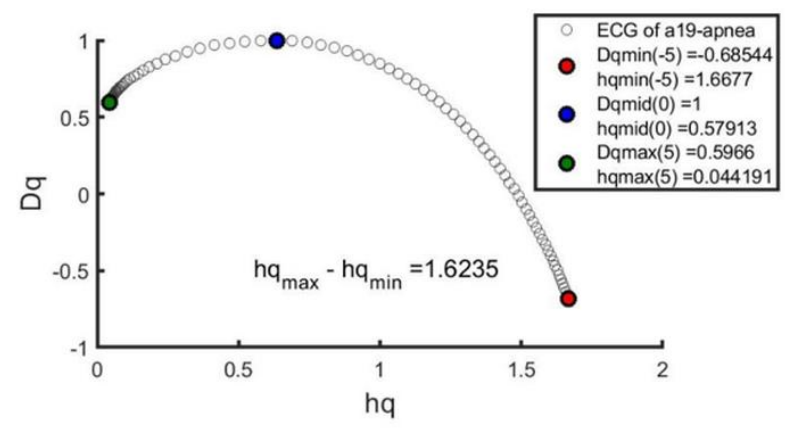

(c)

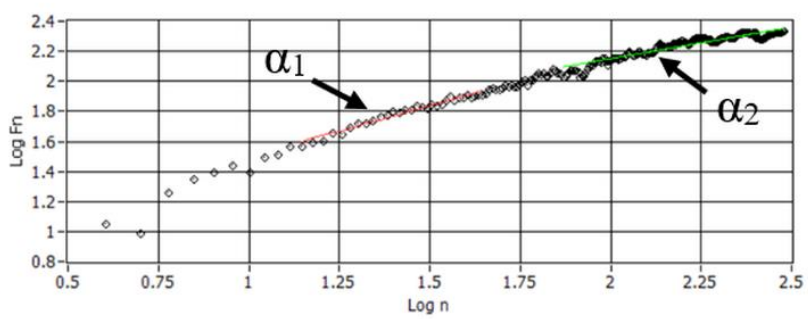

(b)

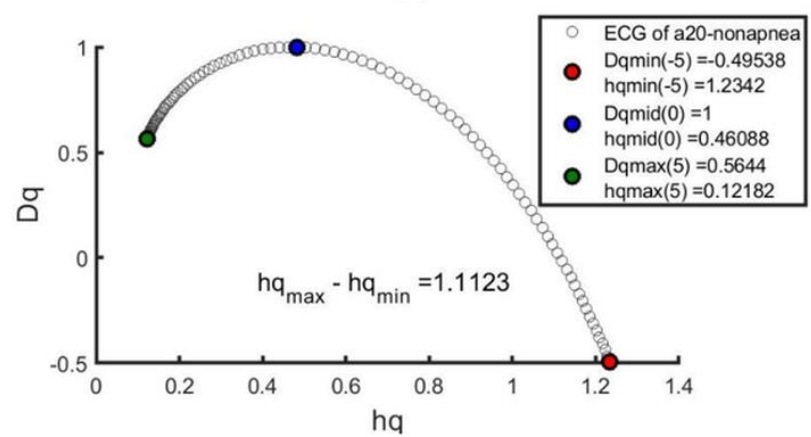

(d)

Figure. 7 The fractal analysis based on self-similarity of ECG signal behaviour: (a) shows linear fitting for apnea event, (b) show linear fitting for non-apnea event using the monofractal approach, (c) show multifractal spectrum for apnea event, and (d) show multifractal spectrum for non-apnea event using the multifractal approach 


\subsection{Analysis of multifractal spectrum based MFDFA}

This section describes MFDFA to the analysis of the signal. The MFDFA approach is used to learn more about structure fractals. The multifractal time series have local fluctuations with both extremely small and large magnitudes that are absent in the monofractal time series. Fig. 7 (c) shows trending results to obtain fractal scaling such as Dq and hq on apnea indication profile. The profile has a duration of 14 minutes 59 seconds and which correspond to the segments by a number of samples of 90000 data points. The results confirm that the features have feature value for the parameters Dqmin, hqmin, hqmid, Dqmax, hqmax, hqmaxhqmin is -0.68544 , $1.6677, \quad 0.57913, \quad 0.5966, \quad 0.044191, \quad 1.6235$ respectively. However, Fig. 7 (d) shows the results of fractal scaling for the signal profile with an indication of non-apnea with the number of samples of 72000 data points. The profile has a duration of 11 minutes and 59 seconds in length. The results confirm that the calculation of feature value for parameters Dqmin, hqmin, hqmid, Dqmax, hqmax, hqmaxhqmin is $0.49536,1.2342,0.46088,0.5644,0.12182,1.1123$ respectively. Both of the experiment results explain that the MFDFA method in the signal profile is a reflection of the temporal variation in the structure time domain. These results are visualized in the width and shape of multifractal spectrum. Furthermore, the fractal spectrum has a smaller range that makes the fractal structure more stable, but a broad-spectrum range represents the fractal structure of the slow fluctuation. The results conclude that the multifractal analysis could yield appropriately to investigate fractal scaling in spectrum than the other approaches.

\subsection{Statistical analysis}

The set of features has functions and influences when used for the classification process. The feature capability can be measured by testing its significance level so that the differences between the two classes
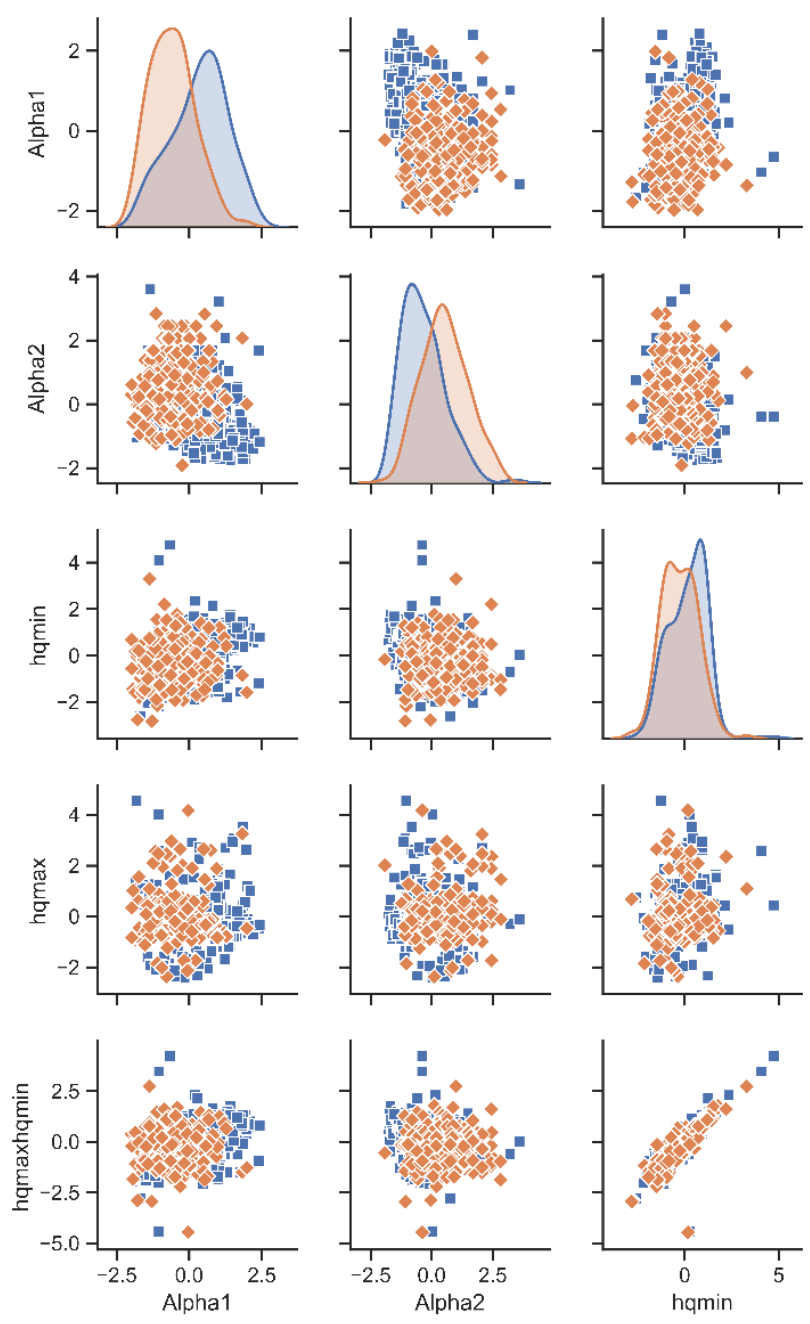
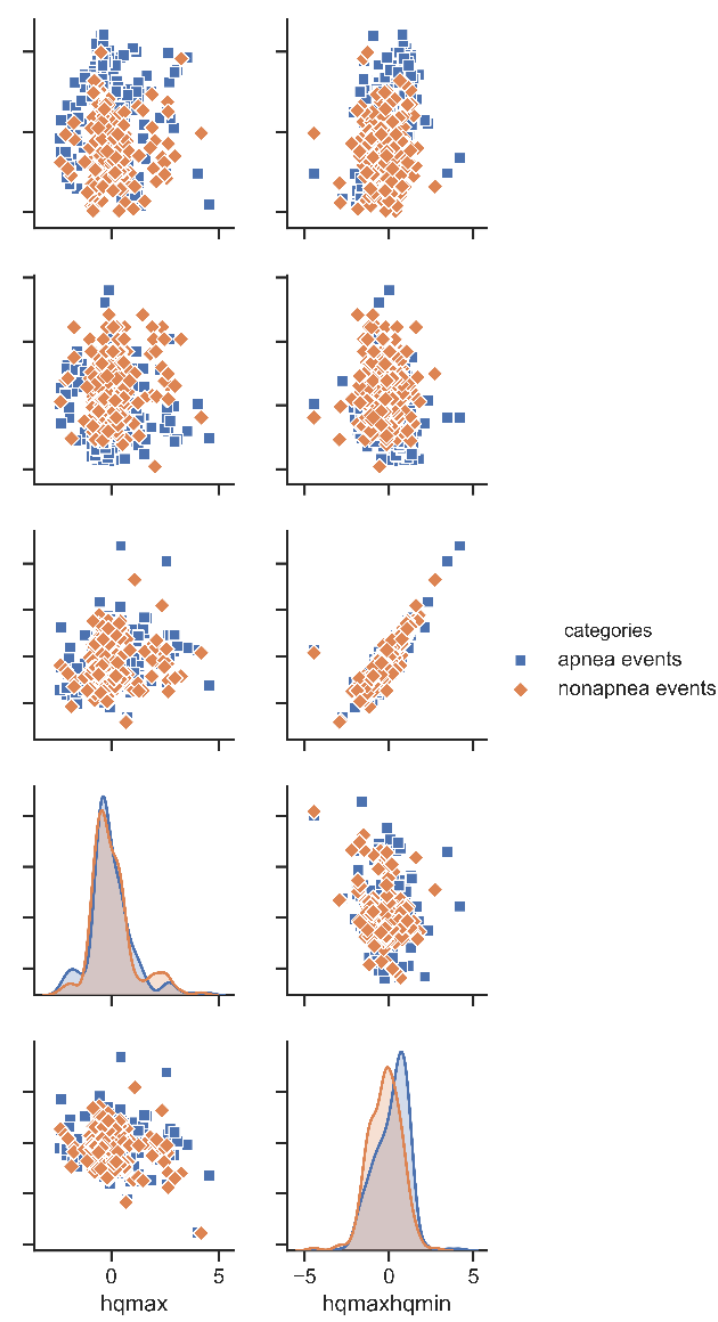

Figure. 8 The graph of the standard normal distribution for selective feature based on z-score 
Table 2. Here are the parameters of various SVM kernels

\begin{tabular}{ll}
\hline Various kernel & Setting parameters \\
\hline default setting of & $\mathrm{C}=1.0$, degree $=3$, \\
kernel: & gamma='auto', coef0=0.0, \\
rbf*, linear, sigmoid & shrinking=True, \\
and polynomial & probability=False, \\
& tolerance=0.001, cache \\
& size=200, class weight=None, \\
& verbose=False, max iteration=- \\
& 1, decision function \\
& shape='ovr', break ties=False, \\
& random state=None
\end{tabular}

can be seen based on the features fed in the input classifier. Ten features obtained from the monofractal feature set (4 features) and multifractal feature set (6 features) were presented with various feature value. They were score on their abilities to identify the pattern of sleep disorder for non-apnea events and apnea events. The scaling fractal with monofractal and multifractal structures is fundamentally different in the study of a signal, as each measures the RMS and determines slopes. One of the differences between the two is that the MFDFA approach is very useful when performing local signal trends that are robust with small and large magnitudes.

In general, significance levels are represented in terms of forms $p<0.05$. The $p$-value is considered as a probability in interpreting the two samples tested. The test results are compared with the null hypothesis as the basis of an assumption. Therefore, if the pvalue is below the assumption, it can be concluded that the feature has a different distribution.

Conversely, However, if the p-value is above the assumption, then the distribution is identical. As mention previously, the results of the p-value distribution are tabulated in Table 1. The results of these experiments confirm that the significance test is known to have significant differences between the two classes. More specifically, this strategy may lead to affect the classification process. Therefore, the distribution of feature values generated at the time of feature extraction needs to be recognized in deeply.

To obtain the normally distributed is a feature scaling by calculating the mean and standard deviation of each feature value. Furthermore, standard deviations are used as standard scores which make a normal distribution yield the standard normal distribution. The preprocessing technique with scaling properties makes every feature has potentially contributed to improving accuracy. To appropriately use this approach, the applied z-score for each feature value is normalized scoring and then calculated as follows:

$$
z=\frac{x-\mu}{\sigma}
$$

Meanwhile, $z$ is the standard score, $x$ denotes the value to be standardized, $\mu$ is mean, and $\sigma$ denotes the standard deviation.

Fig. 8 shows the result of the standard normal distribution using z-score for the selective feature, i.e., alpha1, alpha2, hqmin, hqmax, and hqmaxhqmin. It can be seen that if a frequency distribution is normally distributed, the probability of a score occurring by standardizing the scores can be calculated. The fact is that the results of testing using the z-scores is very useful in making decisions about the distribution of datasets. Therefore, the characteristics of each feature set in generating feature values significantly affect the distribution of datasets in each class. The significance of the differences in each class determines the success in each dataset training process to get the desired optimization model.

\subsection{SVM classifier and performance evaluation}

In recent years, the development of SVM has been supported by many kernels that can be used to obtain hyperplane that matches the characteristics of datasets so that kernel functions can make class separation better and more structured. Table 2 shows some parameter settings for each kernel, and the available options related to the recommended values for getting the model with the best accuracy.

The values of SVM kernel parameters are based on the optimizing training model. In this study, we trained our model using a training set to fit the optimization parameters by $90 \%$. Then for tuning the parameters, we used the validation set by $20 \%$ out of $90 \%$ of the training set, and used a testing set to assess the performance of the classifier by $10 \%$.

The specifics of the classification performance obtained using various SVM kernels are presented in Table 4. Three measures, i.e., accuracy, sensitivity, and specificity, were used to assess the performance

Table 3. Improving hyperparameters of SVM RBF kernel

\begin{tabular}{|c|c|c|c|c|c|}
\hline \multicolumn{6}{|c|}{ Improving hyperparameters for SVM RBF Kernel } \\
\hline \multicolumn{3}{|c|}{ Low to medium } & \multicolumn{3}{|c|}{ Medium to high } \\
\hline gamma & $\mathbf{C}$ & $\begin{array}{l}\text { Acc } \\
(\%)\end{array}$ & gamma & $\mathbf{C}$ & $\begin{array}{l}\text { Acc } \\
(\%)\end{array}$ \\
\hline 0.001 & 0.001 & 78.43 & 1 & 1 & 92.16 \\
\hline 0.001 & 0.01 & 64.71 & 1 & 10 & 90.20 \\
\hline 0.001 & 0.1 & 68.63 & 1 & 100 & 82.35 \\
\hline 0.01 & 0.001 & 72.55 & 10 & 1 & 66.67 \\
\hline 0.01 & 0.01 & 70.59 & 10 & 10 & 62.75 \\
\hline 0.01 & 0.1 & 66.67 & 10 & 100 & 74.51 \\
\hline 0.1 & 0.001 & 76.47 & 100 & 1 & 68.63 \\
\hline 0.1 & 0.01 & 72.55 & 100 & 10 & 56.86 \\
\hline 0.1 & 0.1 & 76.47 & 100 & 100 & 64.71 \\
\hline
\end{tabular}

International Journal of Intelligent Engineering and Systems, Vol.14, No.2, 2021 
Table 4. Performance comparison of various SVM kernels for classification

\begin{tabular}{lccc}
\hline Methods & $\begin{array}{c}\text { sensitivity } \\
(\boldsymbol{\%})\end{array}$ & $\begin{array}{c}\text { specificity } \\
(\boldsymbol{\%})\end{array}$ & $\begin{array}{c}\text { accuracy } \\
(\boldsymbol{\%})\end{array}$ \\
\hline $\begin{array}{l}\text { SVM } \\
\text { polynomial }\end{array}$ & 93.75 & 72.94 & 76.24 \\
SVM & 84.21 & 77.78 & 80.20 \\
sigmoid & & & \\
SVM linear & 76.74 & 89.66 & 84.16 \\
SVM RBF & 88.24 & 94.12 & 92.16 \\
\hline
\end{tabular}

of the SVM classifier. The classification result is observed that the SVM with RBF kernel achieved the highest classification accuracy of $92.16 \%$ and the highest specificity of $94.12 \%$. The SVM with polynomial kernel achieved a sensitivity of $93.75 \%$.

The gamma and $\mathrm{C}$ parameter settings are to reduction errors, limit the possibility of overfitting and determine curvature weight of the decision boundary to obtained the good margins. Table 3 shows that the higher the $\mathrm{C}$ value, the lower the misclassification rate so that the model accuracy increases. Conversely, a smaller value of $\mathrm{C}$ results in a larger margin of error and consequences in lower accuracy. However, at one particular position the specific value of $\mathrm{C}$ yields the best accuracy. In addition, a lower value gamma setting will result in a lower accuracy model. The same thing occurs if the gamma value is set higher resulting in a model with lower accuracy. This means that the gamma value will provide a model with good decision boundaries. It can be noticed from the Table 3 that the combination gamma and $\mathrm{C}$ parameter revealed a higher accuracy $(92.16 \%, 90.20 \%, 82.35 \%)$ at the best gamma $=1$ and $\mathrm{C}=1$, gamma $=1$ and $\mathrm{C}=10$, gamma $=1$ and $\mathrm{C}=100$, respectively. More specifically, the trainable parameters of the RBF kernel are optimization parameters with the optimization setting of $\mathrm{C}=1.0$, and gamma $=1.0$.

As the appropriate model is being tested on the testing set, the SVM applied to the single lead of ECG signal had an average sensitivity of $85.74 \% \pm 7.16 \%$, specificity of $83.63 \% \pm 9.91 \%$, and accuracy of $83.19 \% \pm 6.79 \%$. In order to implemented, the specificity parameter is the most important metric because it measures the ability of the algorithm to detect apnea patients. It does not classify a patient as non-apnea when they are apnea.

The confusion matrix observed the SVM that uses the RBF kernel has the best classification accuracy and specificity, among others of $92.16 \%$ and, $94.12 \%$, respectively. Some of these differences are described by the comparison between the kernels. The difference between kernel affects accuracy. The accuracy of the linear kernel, sigmoid kernel, and the polynomial kernel is reduced by $8 \%, 11.96 \%$, and $15.92 \%$, respectively, compared by the RBF kernel. Therefore, it is clear from the Table 4 that an RBF kernel outperforms the other kernels. Related to the calibration curve in Fig. 9, it shows the predicted probabilities on SVM linear and SVM RBF was appropriate in line with perfectly calibrated. The result that both obtained the optimal values of accuracy. Conversely, the SVM sigmoid kernel and SVM polynomial have the worst predicted probabilities with accuracy lower than other kernels of $80.20 \%$ and 76.24 , respectively.

\section{Discussion}

The main aim of this study is to improve sleep disorders in real time for reducing visual inspection and manual evaluation. It is because visual examination takes time and acceptance discrepancies rely on the skills of the medical employee. To carry out an in-depth description on preprocessing stage, feature extraction stage, and classification method, this study more exploits sleep scoring with the examination single lead ECG with complexity and robustness in more than ten minutes length. The new features are proposed to boost the efficiency of the algorithm for classification to the obtained acceptable pattern for clear distinctions between apnea and nonapnea events based on trending signal behaviour and characteristic of QRS morphologies using fractal analysis. The last one is to attain proper optimal parameter to improve the large margin of SVM hyperplane. As described in the previous section, it is obvious that solving the sleep disorder classification case using the feature set of fractal analysis has improved performance. This improvement is better than only using the feature set of the variability of time and frequency domain. In addition, the improvement is obtained using only limited features but can provide high differences between the two classes so that the algorithm works more optimally. A performance comparison with respect for the existing literatures that report various works for classification on apnea ECG physionet is presented in Table 5.

In order to describe in more depth of the proposed approach, the scientific contributions of this study are described. The scientific contributions of this research for effective involvement, we used nonoverlapping of ECG from single lead with a recording duration of more than ten minutes per event, the number of features used for the classification process was not more than ten features, the assessment process of ECG records based on the trend of a signal and complex QRS wave morphology. Other scientific 
contributions are parameter optimization to improve the SVM method with RBF kernel and produce an automatic sleep disturbance detection system that reduces human intervention during visual inspection and manual assessment. These contributions will be described further in another section. In addition, the results can be compared with other approaches or methods for detecting sleep disorders applied to the Apnea ECG-database. The comparison of the results is as shown in Table 5 in the form of the number of features, the classification method used and the resulting performance measures.

The first and second contribution are related to the number of features and the feature extraction method chosen. As previously stated, ECG recording analysis for patients with indications of sleep disorder is widely used for assessment. One of the most widely used diagnostic tools is based on the calculation of heart rate variability [27] with 28 features that represent the variability of power in mean and standard deviation. In addition, through HRV, many features can be used such as analysis of the energy and fuzzy entropy feature of intrinsic band function with total features of 12 and 1-minute length of the segment [8]. The results of the SVM kernel RBF classification in this study are sensitivity, specificity, and accuracy of $79.70 \%, 73.35 \%$ and $76.58 \%$, respectively. The results of the performance evaluation are quite promising and can still be improved by other approaches. The other studies with a higher number of features, 32 features derived from the combination of time domain analysis of HRV, ECG respiration, and cardiopulmonary coupling in one-minute length from single lead of ECG [28]. Their results confirm that the combination approach presented an accuracy of $89.8 \%$, the specificity of $92.9 \%$, and the sensitivity of $84.7 \%$. The performance measures have improved compared to previous related research. However, with more features analysed, it will have an impact on the complexity of the computational process, allow the loss of clinical information, and does not represent all the information related to the pattern of ECG signal for the sleep disorder. The other studies have presented to obtain performance improvements, the method utilized deep learning to learn feature based 5 criteria. Finally, a decision fusion method was quite successful with sensitivity, specificity, and accuracy of $88.9 \%, 82.1 \%$ and $84.7 \%$ respectively [29]. Other studies with significant improvements in the three evaluation parameters were found to be in the range above $90 \%$ [30]. This improvement is not only in the extraction method but also in the preprocessing stage with reducing the error of the result of RR interval detection using a local median filter modification orthogonal wavelet to employing 12 feature set [5]. Correspondently, the average accuracy, average
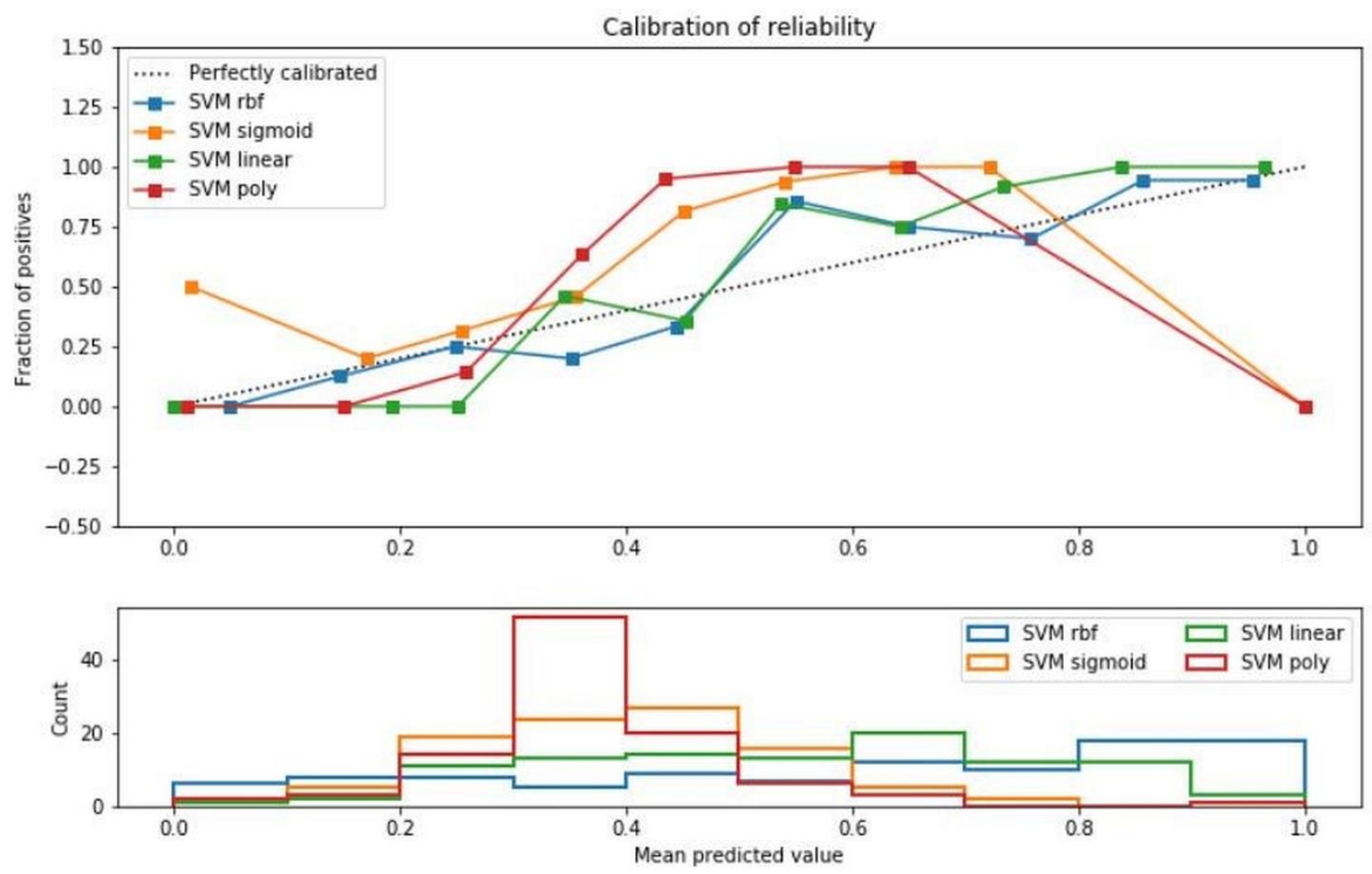

Figure. 9 A reliability diagram for observed relative frequency and the predicted probabilities 
Table 5. Performance comparison of various works on classification of apnea ECG physionet

\begin{tabular}{|c|c|c|c|c|c|}
\hline $\begin{array}{c}\text { Authors } \\
\text { (reference) }\end{array}$ & $\begin{array}{c}\text { Number } \\
\text { of } \\
\text { features }\end{array}$ & Decision & Classification method & $\begin{array}{c}\text { Accuracy } \\
(\%)\end{array}$ & others \\
\hline Ngyuyen et al [32] & 33 & apnea/hypopnea & Decision fusion & 84.19 & N/A \\
\hline Varon et al [31] & 28 & apnea/hypopnea & $\begin{array}{l}\text { LS-SVM Classifier using } \\
\text { RBF kernel }\end{array}$ & 84.74 & N/A \\
\hline Hassan [14] & 36 & apnea/normal & AdaBoost & 87.33 & $\begin{array}{l}\text { Sensitivity: } \\
81.99 \% \\
\text { specificity: } \\
90.72 \%\end{array}$ \\
\hline Hassan et al [15] & N/A & apnea/normal & RUSBoost & 88.88 & $\begin{array}{l}\text { Sensitivity: } \\
87.58 \% \text {; } \\
\text { specificity: } \\
91.49 \%\end{array}$ \\
\hline Chen et al [30] & 18 & apnea/normal & Kernel density classifier & 82.07 & $\begin{array}{l}\text { Sensitivity: } \\
83.23 \% \\
\text { specificity: } \\
80.24 \%\end{array}$ \\
\hline Sharma et al [5] & 12 & apnea/normal & Gaussian SVM & $\begin{array}{l}90.87 \\
\text { (average) }\end{array}$ & $\begin{array}{l}\text { Average } \\
\text { Sensitivity: } \\
92.43 \% \text {; } \\
\text { Average } \\
\text { specificity: } \\
88.33 \%\end{array}$ \\
\hline Li et al [29] & 5 & OSA/normal & $\begin{array}{l}\text { Decision Fusion with } 5 \\
\text { criteria features }\end{array}$ & 84.7 & $\begin{array}{l}\text { Sensitivity: } \\
88.9 \% \\
\text { specificity: } \\
82.1 \%\end{array}$ \\
\hline our work & 10 & apnea/non-apnea & SVM-RBF & 92.16 & $\begin{array}{l}\text { Sensitivity: } \\
88.24 \% \\
\text { specificity: } \\
94.12 \%\end{array}$ \\
\hline
\end{tabular}

sensitivity and average specificity are $90.87 \%$, $92.43 \%$, and $88.33 \%$ respectively.

To explain the third contribution, fractal analysis based on monofractal and multifractal analyses are frequently adopted to nonlinear behaviour of ECG signals. However, the application is still individually used to solve cases related to the fractal structure in the presence of dynamic forms. However, monofractal analysis techniques have been used for a long time [19]. However, its application is still reliable to solve problems related to correlation in time series with the nonlinear phenomenon. In the case of detection or classification of sleep disorders, the use of extraction methods with investigative techniques using fractal analysis, especially in monofractal analysis alone or in combination, has never existed. The previous studies have focused more on analytical approaches based on timefrequency domains and their derivatives [28,31]. The characteristics of sleep disorder are not only recognized by the pattern based on the occurrence of ECG wave morphology, i.e., p wave, QRS wave, and $\mathrm{t}$ wave. However, we are able to identify the periodic pattern of sleep disorder through signal behaviour based on dynamism so that the resulting parameters can be obtained and calculated. In the present study, we attempted to combine monofractal and multifractal analyses to explore the dynamic of the ECG signal by examining the obtained feature set for the diagnosis of sleep disorder. The experimental results are very clear that the two approaches can 
recognize signal patterns and result in improved performance of the classification method.

A whole other contribution is how the optimization effects for various kernels on the SVM improve the classification efficiency methods. Finally, the proposed method comparison can provide other alternatives in producing computerbased tools to minimize visual inspection and manual scoring by medical employees. The results of the experiment show that the problem in the classification process is the distribution of data on apnea events and non-apnea events are not balanced. However, choosing the right classification method is quite able to reduce the effect of the imbalance of the dataset. The facts show that the algorithm is better at detecting both classes with sensitivity and specificity levels in the range above $88 \%$. In general, it could be concluded that the effectiveness of feature sets successfully and effectively exploits the difference of pattern of apnea and non-apnea events, which is deeply complex and its dynamical information changes during sleep.

\section{Conclusion}

In this study, we report the ECG-apnea recordings of the patients with different segmentation for the preprocessing and extraction processes. We employed the monofractal and the multifractal analyses to generate ten different features such as alpha1, residue1, alpha2, residue2, Dqmin, hqmin, hqmid, Dqmax, hqmax, and hqmaxhqmin. The experiment is directed using various classification models in order to find out which one emerges as the best performing. The dominant features are then used for the classification using SVM classifier with different kernels such as RBF kernel, linear kernel, polynomial kernel, and sigmoid kernel. The proposed method that yields the best-performing kernel is the RBF with a classification accuracy of $92.16 \%$, sensitivity of $88.24 \%$, and specificity of $94.12 \%$. The advantage of the study shows that multi-feature analysis with fractal analysis is a capable means to assist a clinical expert in getting potential sleep disorder screening, particularly when applied to home sleep testing independently.

\section{Conflicts of Interest}

The authors declare no conflict of interest.

\section{Author Contributions}

Conceptualization, Mauridhi H. Purnomo, I Ketut Eddy Purnama, and Hideya Takahashi; methodology, Iman Fahruzi; software, Iman Fahruzi; validation, I
Ketut Eddy Purnama and Kayo Yoshimoto; formal analysis, Iman Fahruzi, I Ketut Eddy Purnama and Mauridhi H. Purnomo; writing - original draft preparation, Iman Fahruzi and I Ketut Eddy Purnama; writing - review and editing, Mauridhi H. Purnomo and I Ketut Eddy Purnama; visualization, Iman Fahruzi and I Ketut Eddy Purnama; supervision, I Ketut Eddy Purnama, Kayo Yoshimoto, Hideya Takahashi and Mauridhi H. Purnomo; project administration, Iman Fahruzi; funding acquisition, Iman Fahruzi.

\section{Acknowledgments}

This work was supported by BPPDN scholarship and Enhancing International Publication Program (EIP) scholarship from the Ministry of Research, Technology, and Higher Education - Republic of Indonesia and University Center of Excellence on Artificial Intelligence for Healthcare and Society (UCE AIHeS), Institut Teknologi Sepuluh Nopember, Surabaya. Furthermore, this work was also supported by Politeknik Negeri Batam.

\section{References}

[1] M. J. Sateia, "International Classification of Sleep Disorders-Third Edition", Chest, Vol. 146, No. 5, pp. 1387-1394, 2014.

[2] M. Sharma, D. Goyal, P. V. Achuth, and U. R. Acharya, "An accurate sleep stages classification system using a new class of optimally time-frequency localized three-band wavelet filter bank", Computers in Biology and Medicine, Vol. 98, pp. 58-75, 2018.

[3] R. K. Tripathy and U. Rajendra Acharya, "Use of features from RR-time series and EEG signals for automated classification of sleep stages in deep neural network framework", Biocybernetics and Biomedical Engineering, Vol. 38, No. 4, pp. 890-902, 2018.

[4] H. Yoon, S. H. Hwang, J.-W. Choi, Y. J. Lee, D.-U. Jeong, and K. S. Park, "Slow-Wave Sleep Estimation for Healthy Subjects and OSA Patients Using R-R Intervals", IEEE Journal of Biomedical and Health Informatics, Vol. 22, No. 1, pp. 119-128, 2018.

[5] M. Sharma, M. Raval, and U. R. Acharya, "A new approach to identify obstructive sleep apnea using an optimal orthogonal wavelet filter bank with ECG signals", Informatics in Medicine Unlocked, p. 100170, 2019.

[6] Ş. Yücelbaş, C. Yücelbaş, G. Tezel, S. Özşen, S. Küççüktürk, and Ş. Yosunkaya, "Predetermination of OSA degree using morphological features of the ECG signal”, 
Expert Systems with Applications, Vol. 81, pp. 79-87, 2017.

[7] G. Dorantes-Méndez, M. O. Mendez, A. Alba, L. Parrino, and G. Milioli, "Time-varying analysis of the heart rate variability during A-phases of sleep: Healthy and pathologic conditions", Biomedical Signal Processing and Control, Vol. 40, pp. 111-116, 2018.

[8] R. K. Tripathy, "Application of intrinsic band function technique for automated detection of sleep apnea using HRV and EDR signals", Biocybernetics and Biomedical Engineering, Vol. 38, No. 1, pp. 136-144, 2018.

[9] A. Nishad, R. B. Pachori, and U. R. Acharya, "Application of TQWT based filter-bank for sleep apnea screening using ECG signals", Journal of Ambient Intelligence and Humanized Computing, 2018.

[10] M. Shahbakhti, H. Bagheri, B. Shekarchi, S. Mohammadi, and M. Naji, "A New Strategy for ECG Baseline Wander Elimination Using Empirical Mode Decomposition", Fluct. Noise Lett., Vol. 15, No. 02, p. 1650017, 2016.

[11] P. Gupta, K. K. Sharma, and S. D. Joshi, "Baseline wander removal of electrocardiogram signals using multivariate empirical mode decomposition", Healthcare Technology Letters, Vol. 2, No. 6, pp. 164-166, 2015.

[12] M. Radha, P. Fonseca, A. Moreau, M. Ross, A. Cerny, P. Anderer, X. Long, and R. M. Aarts, "Sleep stage classification from heart-rate variability using long short-term memory neural networks", Scientific Reports, Vol. 9, No. 1, 2019.

[13] N. Banluesombatkul, T. Rakthanmanon, and T. Wilaiprasitporn, "Single Channel ECG for Obstructive Sleep Apnea Severity Detection Using a Deep Learning Approach", in TENCON 2018 - 2018 IEEE Region 10 Conf., pp. 20112016, 2018.

[14] A. R. Hassan and Md. A. Haque, "Computeraided obstructive sleep apnea screening from single-lead electrocardiogram using statistical and spectral features and bootstrap aggregating", Biocybernetics and Biomedical Engineering, Vol. 36, No. 1, pp. 256-266, 2016.

[15] A. R. Hassan and Md. A. Haque, "An expert system for automated identification of obstructive sleep apnea from single-lead ECG using random under sampling boosting", Neurocomputing, Vol. 235, pp. 122-130, 2017.

[16] T. Penzel, G. B. Moody, R. G. Mark, A. L. Goldberger, and J. H. Peter, "The apnea-ECG database", in Computers in Cardiology 2000.
Vol.27 (Cat. 00CH37163), Cambridge, MA, USA, pp. 255-258,2000.

[17] I. Fahruzi, I. K. E. Purnama, and M. H. Purnomo, "An Investigation of Dynamic Features Influence in ECG-Apnea Using Detrended Fluctuation Analysis", in 2018 International Conf. on Intelligent Autonomous Systems (ICoIAS), Singapore, pp. 23-27, 2018.

[18] A. Bunde, S. Havlin, J. W. Kantelhardt, T. Penzel, J. H. Peter, and K. Voigt, "Correlated and uncorrelated regions in heart-rate fluctuations during sleep.", Phys Rev Lett, Vol. 85, No. 17, pp. 3736-3739, 2000.

[19] C. K. Peng, S. Havlin, H. E. Stanley, and A. L. Goldberger, "Quantification of scaling exponents and crossover phenomena in nonstationary heartbeat time series", Chaos, Vol. 5, No. 1, pp. 82-87, 1995.

[20] T. Penzel, J. W. Kantelhardt, L. Grote, J. H. Peter, and A. Bunde, "Comparison of detrended fluctuation analysis and spectral analysis for heart rate variability in sleep and sleep apnea", IEEE Transactions on Biomedical Engineering, Vol. 50, No. 10, pp. 1143-1151, 2003.

[21] E. A. F. Ihlen, "Introduction to Multifractal Detrended Fluctuation Analysis in Matlab", Frontiers in Physiology, Vol. 3, 2012.

[22] J. W. Kantelhardt, S. A. Zschiegner, E. Koscielny-Bunde, S. Havlin, A. Bunde, and H. E. Stanley, "Multifractal detrended fluctuation analysis of nonstationary time series", Physica A: Statistical Mechanics and its Applications, Vol. 316, No. 1, pp. 87-114, 2002.

[23] S. Chatterjee, S. Pratiher, and R. Bose, "Multifractal detrended fluctuation analysis based novel feature extraction technique for automated detection of focal and non-focal electroencephalogram signals", IET Science, Measurement Technology, Vol. 11, No. 8, pp. 1014-1021, 2017.

[24] K. Philippopoulos, N. Kalamaras, C. G. Tzanis, D. Deligiorgi, and I. Koutsogiannis, "Multifractal Detrended Fluctuation Analysis of Temperature Reanalysis Data over Greece", Atmosphere, Vol. 10, No. 6, p. 336, 2019.

[25] X. Tang, X. Yang, and F. Wu, "Multifractal detrended fluctuation analysis parallel optimization strategy based on openMP for image processing", Neural Computing and Applications, 2019.

[26] M. Awad and R. Khanna, "Support Vector Machines for Classification", in Efficient Learning Machines: Theories, Concepts, and Applications for Engineers and System 
Designers, M. Awad and R. Khanna, Eds. Berkeley, CA: Apress, 2015.

[27] A. H. Khandoker, M. Palaniswami, and C. K. Karmakar, "Support Vector Machines for Automated Recognition of Obstructive Sleep Apnea Syndrome from ECG Recordings", IEEE Transactions on Information Technology in Biomedicine, Vol. 13, No. 1, pp. 37-48, 2009.

[28] P. de Chazal and N. Sadr, "Sleep apnoea classification using heart rate variability, ECG derived respiration and cardiopulmonary coupling parameters", In: Proc. of 2016 38th Annual International Conf. of the IEEE Engineering in Medicine and Biology Society $(E M B C), 2016$.

[29] K. Li, W. Pan, Y. Li, Q. Jiang, and G. Liu, “A method to detect sleep apnea based on deep neural network and hidden Markov model using single-lead ECG signal", Neurocomputing, Vol. 294, pp. 94-101, 2018.

[30] L. Chen, X. Zhang, and H. Wang, "An Obstructive Sleep Apnea Detection Approach Using Kernel Density Classification Based on Single-Lead Electrocardiogram", Journal of Medical Systems, Vol. 39, No. 5, 2015.

[31] C. Varon, A. Caicedo, D. Testelmans, B. Buyse, and S. V. Huffel, "A Novel Algorithm for the Automatic Detection of Sleep Apnea from Single-Lead ECG', IEEE Transactions on Biomedical Engineering, Vol. 62, No. 9, pp. 2269-2278, 2015.

[32] H. D. Nguyen, B. A. Wilkins, Q. Cheng, and B. A. Benjamin, "An Online Sleep Apnea Detection Method Based on Recurrence Quantification Analysis", IEEE Journal of Biomedical and Health Informatics, Vol. 18, No. 4, pp. 1285-1293, 2014. 